This is a preprint of: "Spatial convex but non-strictly convex double-pyramidal central configurations of the $(2 \mathrm{n}+2)$-body problem", Montserrat Corbera, Jaume Llibre, J. Dyn. Diff. Equat., 2019.

DOI: $[10.1007 / \mathrm{s} 10884-019-09798-3]$

\title{
Spatial convex but non-strictly convex double-pyramidal central configurations of the $(2 n+2)$-body problem
}

\author{
Montserrat Corbera ${ }^{1}$ and Jaume Llibre ${ }^{2}$ \\ ${ }^{1}$ Departament de Tecnologies Digitals i de la Informació, Universitat de Vic, \\ 08500 Vic, Barcelona, Spain. E-mail: montserrat.corbera@uvic.cat \\ 2 Departament de Matemàtiques, Universitat Autònoma de Barcelona, \\ 08193 Bellaterra, Barcelona, Spain. E-mail: jllibre@mat.uab.cat
}

\begin{abstract}
A configuration of the $N$ bodies is convex if the convex hull of the positions of all the bodies in $\mathbb{R}^{3}$ does not contain in its interior any of these bodies. And a configuration is strictly convex if the convex hull of every subset of the $N$ bodies is convex.

Recently some authors have proved the existence of convex but non-strictly convex central configurations for some $N$-body problems. In this paper we prove the existence of a new family of spatial convex but non-strictly convex central configurations of the $(2 n+2)$-body problem.

More precisely, we prove that for $n \geqslant 4$ there are spatial convex but non-strictly convex central configurations of the $(2 n+2)$-body problem consisting of $n$ masses equal to $m_{1}$ at the vertices of a regular $n$-gon, $n$ masses equal to $m_{2}$ at the vertices of the $n$-gon whose vertices are the midpoints of the edges of the initial $n$-gon, and two masses equal to $m_{3}$ on the straight line orthogonal to the plane containing the two $n$-gons passing through their barycenters. Moreover, we show that for $n=3$ does not exist such spatial convex but nonstrictly convex central configurations. Note that the convex hull of such central configurations is formed by two equal pyramides with
\end{abstract}


the base formed by the big $n$-gon, glued by their bases. So we call them spatial convex but non-strictly convex double-pyramidal central configurations of the $2 n+2$-body problem.

\section{Introduction and statement of the main result}

The equations of motion of the spatial $N$-body problem are

$$
m_{k} \ddot{\mathbf{q}}_{k}=-\sum_{\substack{j=1 \\ j \neq k}}^{N} G m_{k} m_{j} \frac{\mathbf{q}_{k}-\mathbf{q}_{j}}{\left|\mathbf{q}_{k}-\mathbf{q}_{j}\right|^{3}},
$$

for $k=1, \ldots, N$, where $G$ is the gravitational constant which will be taken equal to one by choosing conveniently the unit of time, $\mathbf{q}_{k} \in \mathbb{R}^{3}$ is the position vector of the punctual mass $m_{k}$ in an inertial coordinate system, and the two dots denote the second derivative with respect to the time $t$.

The configuration space of the spatial $N$-body problem is

$$
\mathcal{E}=\left\{\left(\mathbf{q}_{1}, \ldots, \mathbf{q}_{N}\right) \in \mathbb{R}^{3 N}: \mathbf{q}_{k} \neq \mathbf{q}_{j}, \text { for } k \neq j\right\} .
$$

Given $N$ positive masses $m_{1}, \ldots, m_{N}$ a configuration $\left(\mathbf{q}_{1}, \ldots, \mathbf{q}_{N}\right) \in \mathcal{E}$ is central if there exists a positive constant $\lambda$ such that

$$
\ddot{\mathbf{q}}_{k}=-\lambda\left(\mathbf{q}_{k}-\mathbf{c m}\right),
$$

for $k=1, \ldots, N$, where

$$
\mathbf{c m}=\frac{\sum_{k=1}^{N} m_{k} \mathbf{q}_{k}}{\sum_{k=1}^{N} m_{k}} .
$$

is the center of mass of the system. Consequently a central configuration $\left(\mathbf{q}_{1}, \ldots, \mathbf{q}_{N}\right) \in \mathcal{E}$ of the $N$-body problem with masses $m_{1}, \ldots, m_{N}$ is a solution of the system of equations

$$
\sum_{\substack{j=1 \\ j \neq k}}^{N} m_{j} \frac{\mathbf{q}_{k}-\mathbf{q}_{j}}{\left|\mathbf{q}_{k}-\mathbf{q}_{j}\right|^{3}}=\lambda\left(\mathbf{q}_{k}-\mathbf{c m}\right),
$$

for $k=1, \ldots, N$ and for some $\lambda$. 
We do not know the first author which shown that $N$ equal masses located at the vertices of a regular $N$-gon is a central configuration of the $N$-body problem for all $N \geqslant 2$.

If we put equal masses at the vertices of any regular polyhedron, then we obtain a spatial central configuration of the $N$-body problem with $N$ equal to the number of vertices (see [2]).

Other spatial central configurations of the $N$-body problem are the pyramidal central configurations, which consist of $N=n+1$ masses, with $n$ equal masses at the vertices of a regular $n$-gon and the $(n+1)$ th mass in the orthogonal straight line to the plane containing the $n$-gon and passing through its barycenter (see for instance [5] and [18]).

Another simple spatial central configurations are the double pyramidal central configurations, which consist of $N=n+2$ masses, $n$ equal masses at the vertices of a regular $n$-gon and the other two equal masses symmetrically localized with respect to the plane containing the $n$-gon on the orthogonal straight line to this plane and passing through its barycenter (see $[8,9,10,11,12,13,14,19,20]$ ). In [16] the authors study these previous central configurations adding a new mass on the barycenter of the $n$-gon. In [4] we studied these double pyramidal central configurations but when the two masses on the orthogonal straight line to the plan containing the $n$-gon through its barycenter are not equal, we called such central configurations by bi-pyramidal central configurations.

We recall that a configuration of the $N$ bodies is convex if the convex hull of the positions of all the bodies in $\mathbb{R}^{3}$ does not contain in its interior any of these bodies. And a configuration is strictly convex if the convex hull of every subset of the $N$ bodies is convex.

As far as we know the first example of a convex but non-strictly convex central configuration appeared in 2010 in the paper [7] of Gidea and Llibre for the planar 5-body problem. In 2012 Chen and Hsiao [3] reproved this previous result of the planar 5-body problem and provided a spatial convex but not strictly convex central configuration for the 7-body problem. In these two first examples of convex but non-strictly convex central configurations three of the bodies are on a straight line. More recently in 2018 Fernandes, Garcia and Mello provided convex but non-strictly convex central configuration for the planar 8-body problem, and for the spatial 10- and 20-body problem. We think that these are all the know convex but non-strictly convex central configuration which appear until now in the literature.

Inspired in the convex but non-strictly convex central configura- 
tion for the spatial 10-body problem found by Fernandes, Garcia and Mello in this paper we shall prove the existence of convex but nonstrictly convex central configuration for the spatial $(2 n+2)$-body problem for all $n \geqslant 4$. More precisely, we prove that for $n \geqslant 4$ there are spatial convex but non-strictly convex central configurations of the $(2 n+2)$-body problem consisting of $n$ masses equal to $m_{1}=1$ (we choose the unit of mass equal to $m_{1}$ ) at the vertices of a regular $n$-gon, $n$ masses equal to $m$ at the vertices of the $n$-gon whose vertices are the midpoints of the edges of the initial $n$-gon, and two masses equal to $\mu$ on the straight line orthogonal to the plane containing the two $n$-gons passing through their barycenters. Moreover, we show that for $n=3$ does not exist such spatial convex but non-strictly convex central configurations. Note that the convex hull of such central configurations is formed by two equal pyramides with the base formed by the big $n$-gon, glued by their bases. So we call them spatial convex but non-strictly convex double-pyramidal central configurations of the $2 n+2$-body problem. Thus our main result is the following one.

Theorem 1 The following statements hold for the central configurations of the $(2 n+2)$-body problem consisting of $n$ masses equal to 1 at the vertices of a regular $n$-gon inscribed in a circle of radius $1, n$ masses equal to $m$ at the vertices of the $n$-gon whose vertices are the midpoints of the edges of the initial n-gon, and two masses equal to $\mu$ on the straight line orthogonal to the plane containing the two n-gons passing through their barycenters and at a distance a of it.

(a) For $n=3$ there does not exist central configurations of this type.

(b) For each $n \geqslant 4$ there exists at least one family of central configurations of this type depending on the parameter a.

Statements (b) and (a) of Theorem 1 are proved in sections 3 and 4 , respectively.

We note that the spatial convex but non-strictly convex central configuration found by Fernandes, Garcia and Mello for the 10-body problem is the central configuration of Theorem 1 for $n=4$.

In section 2 we provide the explicit equations for the doublepyramidal central configurations of the $2 n+2$-body problem. 


\section{Equations of the central configura- tions}

We consider $N=2 n+2$ with $n \in \mathbb{N}, n \geqslant 3, n$ equal masses $m_{1}=\cdots=$ $m_{n}$ at the vertices of a (regular) $n$-gon, $m_{n+1}=\cdots=m_{2 n}=m$ at the midpoint (regular) $n$-gon (the $n$-gon whose vertices are the midpoints of the edges of the initial $n$-gon), and 2 masses $m_{2 n+1}=m_{2 n+2}=\mu$ located symmetrically on the straight line orthogonal to the plane containing the $n$-gons passing through their center. Without loss of generality we can choose the unit of mass so that $m_{1}=\cdots=m_{n}=1$, and we can take the unit of length so that the radius of the circle containing the initial $n$-gon be one. By using complex coordinates in the plane that contains the regular $n$-gons, the positions of the vertices of the initial $n$-gon can be written as $\mathbf{q}_{j}=\left(e^{i \varphi_{j}}, 0\right) \in \mathbb{C} \times \mathbb{R}$ with $\varphi_{j}=2 \pi j / n$ for $j=1, \ldots, n$ and the vertices of the midpoint $n$-gon can be written as $\mathbf{q}_{j+n}=\alpha\left(e^{i\left(\varphi_{j}-\pi / n\right)}, 0\right) \in \mathbb{C} \times \mathbb{R}$ with $\alpha=\cos (\pi / n)$ for $j=1, \ldots, n$. Let the positions of the masses $m_{2 n+1}$ and $m_{2 n+2}$ be $\mathbf{q}_{2 n+1}=(0, a)$ and $\mathbf{q}_{2 n+2}=(0,-a)$, respectively, with $a>0$.

It is easy to check that the center of mass of the system is at the origin. The first $n$ equations of (1) become

$$
\sum_{\substack{j=1 \\ j \neq k}}^{n} \frac{\mathbf{q}_{k}-\mathbf{q}_{j}}{\left|\mathbf{q}_{k}-\mathbf{q}_{j}\right|^{3}}+m \sum_{j=1}^{n} \frac{\mathbf{q}_{k}-\mathbf{q}_{j+n}}{\left|\mathbf{q}_{k}-\mathbf{q}_{j+n}\right|^{3}}+\mu \sum_{\ell=1}^{2} \frac{\mathbf{q}_{k}-\mathbf{q}_{2 n+\ell}}{\left|\mathbf{q}_{k}-\mathbf{q}_{2 n+\ell}\right|^{3}}=\lambda \mathbf{q}_{k},
$$

for $k=1, \ldots, n$, the following $n$ equations become

$$
\sum_{j=1}^{n} \frac{\mathbf{q}_{k+n}-\mathbf{q}_{j}}{\left|\mathbf{q}_{k+n}-\mathbf{q}_{j}\right|^{3}}+m \sum_{\substack{j=1 \\ j \neq k}}^{n} \frac{\mathbf{q}_{k+n}-\mathbf{q}_{j+n}}{\left|\mathbf{q}_{k+n}-\mathbf{q}_{j+n}\right|^{3}}+\mu \sum_{\ell=1}^{2} \frac{\mathbf{q}_{k+n}-\mathbf{q}_{2 n+\ell}}{\left|\mathbf{q}_{k+n}-\mathbf{q}_{2 n+\ell}\right|^{3}}=\lambda \mathbf{q}_{k+n}
$$

for $k=1, \ldots, n$, and the last 2 equations of (1) are

$$
\begin{aligned}
& \sum_{j=1}^{n} \frac{\mathbf{q}_{2 n+1}-\mathbf{q}_{j}}{\left|\mathbf{q}_{2 n+1}-\mathbf{q}_{j}\right|^{3}}+m \sum_{j=1}^{n} \frac{\mathbf{q}_{2 n+1}-\mathbf{q}_{j+n}}{\left|\mathbf{q}_{2 n+1}-\mathbf{q}_{j+n}\right|^{3}}+\mu \frac{\mathbf{q}_{2 n+1}-\mathbf{q}_{2 n+2}}{\left|\mathbf{q}_{2 n+1}-\mathbf{q}_{2 n+2}\right|^{3}}=\lambda \mathbf{q}_{2 n+1}, \\
& \sum_{j=1}^{n} \frac{\mathbf{q}_{2 n+2}-\mathbf{q}_{j}}{\left|\mathbf{q}_{2 n+2}-\mathbf{q}_{j}\right|^{3}}+m \sum_{j=1}^{n} \frac{\mathbf{q}_{2 n+2}-\mathbf{q}_{j+n}}{\left|\mathbf{q}_{2 n+2}-\mathbf{q}_{j+n}\right|^{3}}+\mu \frac{\mathbf{q}_{2 n+2}-\mathbf{q}_{2 n+1}}{\left|\mathbf{q}_{2 n+2}-\mathbf{q}_{2 n+1}\right|^{3}}=\lambda \mathbf{q}_{2 n+2} .
\end{aligned}
$$


It is easy to check that for $j, k=1, \ldots, n$ and $\ell=1,2$

$$
\begin{aligned}
& \left|\mathbf{q}_{k}-\mathbf{q}_{j}\right|=\left|e^{i \varphi_{k}}-e^{i \varphi_{j}}\right|=\sqrt{2} \sqrt{1-\cos \left(\frac{2 \pi(j-k)}{n}\right)}, \\
& \left|\mathbf{q}_{k}-\mathbf{q}_{j+n}\right|=\left|e^{i \varphi_{k}}-\alpha e^{i\left(\varphi_{j}-\pi / n\right)}\right|=\sqrt{1+\alpha^{2}-2 \alpha \cos \left(\frac{2 \pi(j-k)}{n}-\frac{\pi}{n}\right)}, \\
& \left|\mathbf{q}_{k+n}-\mathbf{q}_{j}\right|=\left|\alpha e^{i\left(\varphi_{k}-\pi / n\right)}-e^{i \varphi_{j}}\right|=\sqrt{1+\alpha^{2}-2 \alpha \cos \left(\frac{2 \pi(j-k)}{n}+\frac{\pi}{n}\right)}, \\
& \left|\mathbf{q}_{k+n}-\mathbf{q}_{j+n}\right|=\alpha\left|e^{i\left(\varphi_{k}-\pi / n\right)}-e^{i\left(\varphi_{j}-\pi / n\right)}\right|=\alpha\left|e^{i \varphi_{k}}-e^{i \varphi_{j}}\right|, \\
& \left|\mathbf{q}_{k}-\mathbf{q}_{2 n+\ell}\right|=\sqrt{1+a^{2}}, \\
& \left|\mathbf{q}_{k+n}-\mathbf{q}_{2 n+\ell}\right|=\sqrt{\alpha^{2}+a^{2}}, \\
& \left|\mathbf{q}_{2 n+1}-\mathbf{q}_{2 n+2}\right|=2 a .
\end{aligned}
$$

The first two components of the $n$ vectorial equations (2) are

$$
\sum_{\substack{j=1 \\ j \neq k}}^{n} \frac{e^{i \varphi_{k}}-e^{i \varphi_{j}}}{\left|e^{i \varphi_{k}}-e^{i \varphi_{j}}\right|^{3}}+m \sum_{j=1}^{n} \frac{e^{i \varphi_{k}}-\alpha e^{i\left(\varphi_{j}-\pi / n\right)}}{\left|e^{i \varphi_{k}}-\alpha e^{i\left(\varphi_{j}-\pi / n\right)}\right|^{3}}+2 \mu \frac{e^{i \varphi_{k}}}{\left(1+a^{2}\right)^{3 / 2}}=\lambda e^{i \varphi_{k}},
$$

for $k=1, \ldots, n$. By dividing each equation of (5) by $e^{i \varphi_{k}}$ we get

$$
\sum_{\substack{j=1 \\ j \neq k}}^{n} \frac{1-e^{i\left(\varphi_{j}-\varphi_{k}\right)}}{\left|e^{i \varphi_{k}}-e^{i \varphi_{j}}\right|^{3}}+m \sum_{j=1}^{n} \frac{1-\alpha e^{i\left(\varphi_{j}-\varphi_{k}-\pi / n\right)}}{\left|e^{i \varphi_{k}}-\alpha e^{i\left(\varphi_{j}-\pi / n\right)}\right|^{3}}+2 \mu \frac{1}{\left(1+a^{2}\right)^{3 / 2}}=\lambda,
$$

for $k=1, \ldots, n$. The first two components of the $n$ vectorial equations (3) are

$$
\sum_{j=1}^{n} \frac{\alpha e^{i\left(\varphi_{k}-\pi / n\right)}-e^{i \varphi_{j}}}{\mid \alpha e^{i\left(\varphi_{k}-\pi / n\right)}-e^{\left.i \varphi_{j}\right|^{3}}}+m \sum_{\substack{j=1 \\ j \neq k}}^{n} \frac{1}{\alpha^{2}} \frac{e^{i\left(\varphi_{k}-\pi / n\right)}-e^{i\left(\varphi_{j}-\pi / n\right)}}{\left|e^{i \varphi_{k}}-e^{i \varphi_{j}}\right|^{3}}+2 \mu \frac{\alpha e^{i\left(\varphi_{k}-\pi / n\right)}}{\left(\alpha^{2}+a^{2}\right)^{3 / 2}}=\lambda \alpha e^{i\left(\varphi_{k}-\pi / n\right)},
$$

for $k=1, \ldots, n$. By dividing each equation of $(7)$ by $e^{i\left(\varphi_{k}-\pi / n\right)}$ we get

$$
\sum_{j=1}^{n} \frac{\alpha-e^{i\left(\varphi_{j}-\varphi_{k}+\pi / n\right)}}{\left|\alpha e^{i\left(\varphi_{k}-\pi / n\right)}-e^{i \varphi_{j}}\right|^{3}}+m \sum_{\substack{j=1 \\ j \neq k}}^{n} \frac{1}{\alpha^{2}} \frac{1-e^{i\left(\varphi_{j}-\varphi_{k}\right)}}{\left|e^{i \varphi_{k}}-e^{i \varphi_{j}}\right|^{3}}+2 \mu \frac{\alpha}{\left(\alpha^{2}+a^{2}\right)^{3 / 2}}=\lambda \alpha,
$$


for $k=1, \ldots, n$. The first two components of the two vectorial equations (4) become

$$
\sum_{j=1}^{n} \frac{-e^{i \varphi_{j}}}{\left(1+a^{2}\right)^{3 / 2}}+m \sum_{j=1}^{n} \frac{-\alpha e^{i\left(\varphi_{j}-\pi / n\right)}}{\left(\alpha^{2}+a^{2}\right)^{3 / 2}}=0
$$

Since $\sum_{j=1}^{n} e^{i \varphi_{j}}=0$ and consequently $\sum_{j=1}^{n} e^{i\left(\varphi_{j}-\pi / n\right)}=0$, equation (9) is always satisfied.

It is easy to check that the equations corresponding to the third component of the $n$ vectorial equations (2) and (3) are always satisfied. The third component of the two vectorial equations (4), after dividing by $a$, is equivalent to

$$
\frac{n}{\left(1+a^{2}\right)^{3 / 2}}+m \frac{n}{\left(\alpha^{2}+a^{2}\right)^{3 / 2}}+\frac{\mu}{4 a^{3}}=\lambda .
$$

Since for all $k=1, \ldots, n$ the set $\left\{\varphi_{j}-\varphi_{k}\right\}_{j=1, \ldots, n}$ modulus $2 \pi$ is equal to the set $\{2 \pi j / n\}_{j=1, \ldots, n}$ and the set $\left\{\varphi_{j}-\varphi_{k} \pm \pi / n\right\}_{j, k=1, \ldots, n}$ modulus $2 \pi$ is equal to the set $\{2 \pi j / n \pm \pi / n\}_{j=1, \ldots, n}$, the equations (6) (respectively, (8)) are independent of $k$. So it is not restrictive to take $k=n$. On the other hand, since $\sin (2 \pi j / n)=-\sin (2 \pi-2 \pi j / n)$, $\cos (2 \pi j / n)=\cos (2 \pi-2 \pi j / n)$ and $\sin (2 \pi j / n \pm \pi / n)=-\sin (2 \pi-$ $(2 \pi j / n \pm \pi / n)), \cos (2 \pi j / n \pm \pi / n)=\cos (2 \pi-(2 \pi j / n \pm \pi / n))$, the equations corresponding to the imaginary parts of (6) and (8) are always satisfied. The equations corresponding to the real parts of (6) and (8) are equivalent to

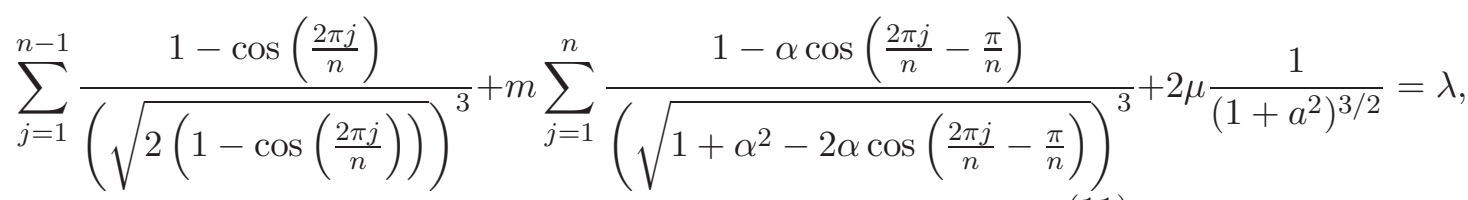

and

$\sum_{j=1}^{n} \frac{\alpha-\cos \left(\frac{2 \pi j}{n}+\frac{\pi}{n}\right)}{\left(\sqrt{1+\alpha^{2}-2 \alpha \cos \left(\frac{2 \pi j}{n}+\frac{\pi}{n}\right)}\right)^{3}}+m \sum_{j=1}^{n-1} \frac{1}{\alpha^{2}} \frac{1-\cos \left(\frac{2 \pi j}{n}\right)}{\left(\sqrt{2\left(1-\cos \left(\frac{2 \pi j}{n}\right)\right)}\right)^{3}}+2 \mu \frac{\alpha}{\left(\alpha^{2}+a^{2}\right)^{3 / 2}}=\lambda \alpha$ 
respectively. Notice that

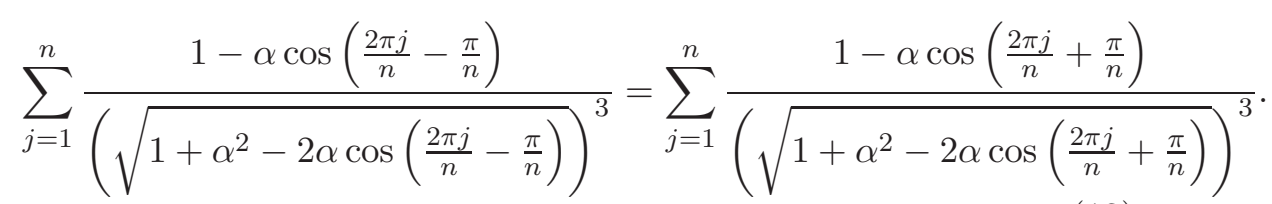

We isolate $\lambda$ from equation (10) and we substitute it into equations (11) and (12). Then, taking into account (13), the equations (1) for the central configurations of our problem are equivalent to the following system of two equations

$$
\left(\begin{array}{ll}
a_{11} & a_{12} \\
a_{21} & a_{22}
\end{array}\right)\left(\begin{array}{c}
m \\
\mu
\end{array}\right)=\left(\begin{array}{l}
-b_{1} \\
-b_{2}
\end{array}\right),
$$

where

$$
\begin{aligned}
a_{11} & =\sum_{j=1}^{n} \frac{1-\alpha \cos \left(\frac{2 \pi j}{n}+\frac{\pi}{n}\right)}{\left(\sqrt{1+\alpha^{2}-2 \alpha \cos \left(\frac{2 \pi j}{n}+\frac{\pi}{n}\right)}\right)^{3}}-\frac{n}{\left(\alpha^{2}+a^{2}\right)^{3 / 2}}, \\
a_{12} & =\frac{2}{\left(1+a^{2}\right)^{3 / 2}}-\frac{1}{4 a^{3}}, \\
a_{21} & =\sum_{j=1}^{n-1} \frac{1}{\alpha^{2}} \frac{1-\cos \left(\frac{2 \pi j}{n}\right)}{\left(\sqrt{2\left(1-\cos \left(\frac{2 \pi j}{n}\right)\right)}\right)^{3}}-\frac{n \alpha}{\left(\alpha^{2}+a^{2}\right)^{3 / 2}}, \\
a_{22} & =\frac{2 \alpha}{\left(\alpha^{2}+a^{2}\right)^{3 / 2}-\frac{\alpha}{4 a^{3}},} \\
b_{1} & =\sum_{j=1}^{n-1} \frac{1-\cos \left(\frac{2 \pi j}{n}\right)}{\left(\sqrt{2\left(1-\cos \left(\frac{2 \pi j}{n}\right)\right)}\right)^{3}}-\frac{n}{\left(1+a^{2}\right)^{3 / 2}}, \\
b_{2} & =\sum_{j=1}^{n} \frac{\alpha-\cos \left(\frac{2 \pi j}{n}+\frac{\pi}{n}\right)}{\left(\sqrt{1+\alpha^{2}-2 \alpha \cos \left(\frac{2 \pi j}{n}+\frac{\pi}{n}\right)}\right)^{3}}-\frac{\alpha n}{\left(1+a^{2}\right)^{3 / 2}} .
\end{aligned}
$$




\section{Proof of statement (b) of Theorem 1}

We start with some auxiliary lemmas. We define

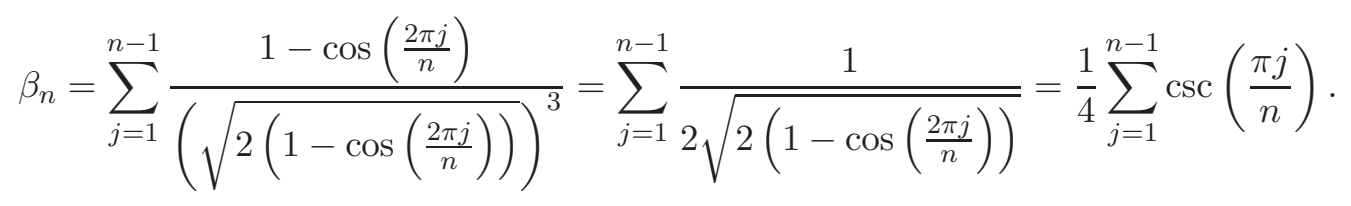

Lemma 2 Let

$$
a^{*}=\sqrt{\left(\frac{n}{\beta_{n}}\right)^{2 / 3}-1},
$$

then the following statements hold.

(a) $a^{*}$ is not defined for $n \geqslant 473$,

(b) for $n<473, b_{1}>0$ when $a>a^{*}$, $b_{1}=0$ when $a=a^{*}$, and $b_{1}<0$ when $a<a^{*}$,

(c) $b_{1}>0$ for $n \geqslant 473$,

(d) $a_{12}>0$ for $a>1 / \sqrt{3}, a_{12}=0$ for $a=1 / \sqrt{3}$, and $a_{12}<0$ for $a<1 / \sqrt{3}$,

(e) $a_{22}>0$ for $a>\alpha / \sqrt{3}, a_{12}=0$ for $a=\alpha / \sqrt{3}$, and $a_{12}<0$ for $a<\alpha / \sqrt{3}$,

(f) $a^{*}>1 / \sqrt{3}$ for $3 \leqslant n<53,0<a^{*}<1 / \sqrt{3}$ for $53 \leqslant n \leqslant 472$.

Proof. In [17] it is proved that $\beta_{n} / n$ is increasing with $n$ and that $\beta_{n} / n>1$ for $n \geqslant 473$. Therefore statement (a) follows directly from the results in [17]. Moreover for $n \geqslant 473$

$$
\frac{b_{1}}{n}=\frac{\beta_{n}}{n}-\frac{1}{\left(1+a^{2}\right)^{3 / 2}}>0,
$$

because $\beta_{n} / n>1$ (see again [17]). This proves statement (c). Statements (b), (d) and (e) follow from simple computations. Finally, computing the values of $a^{*}$ for $n=3, \ldots, 472$ we get statement (f).

Lemma 3 The function $H(n)=\alpha b_{1}-b_{2}$ is positive for all $n \geqslant 473$. 
Independently of us and almost simultaneously Barrabés and Cors in [1] have proved that $H(n)>0$ for all $n \geqslant 5$. Here we give our proof for $n \geqslant 473$ which is different from their proof.

Proof. We define

$$
f(x)=\frac{\alpha(1-\cos x)}{(2(1-\cos x))^{3 / 2}}, \quad F(x)=\frac{\alpha-\cos x}{\left(1+\alpha^{2}-2 \alpha \cos x\right)^{3 / 2}} .
$$

Then $H(n)$ can be written as

$$
H(n)=\sum_{j=1}^{n-1} f\left(\frac{2 \pi j}{n}\right)-\sum_{j=1}^{n} F\left(\frac{2 \pi j}{n}+\frac{\pi}{n}\right) .
$$

Since $\alpha=\cos (\pi / n)$, we have that $F(2 \pi n / n \pm \pi / n)=0$. Therefore

$$
H(n)=\sum_{j=1}^{n-1}\left[f\left(\frac{2 \pi j}{n}\right)-F\left(\frac{2 \pi j}{n}+\frac{\pi}{n}\right)\right] .
$$

On the other hand, since $f(x)=f(2 \pi-x)$ and $F(x)=F(2 \pi-x)$ we have that $H(n)=2 A+A_{o}$ when $n$ is odd and $H(n)=2 A+A_{e}$ when $n$ is even, where

$$
A=\sum_{j=1}^{\left[\frac{n}{2}\right]-1}\left[f\left(\frac{2 \pi j}{n}\right)-F\left(\frac{2 \pi j}{n}+\frac{\pi}{n}\right)\right],
$$

$\left[\frac{n}{2}\right]$ denotes the integer part of $\frac{n}{2}$ and

$$
\begin{aligned}
& A_{o}=2 f\left(\frac{2 \pi\left[\frac{n}{2}\right]}{n}\right)-F\left(\frac{2 \pi\left[\frac{n}{2}\right]}{n}+\frac{\pi}{n}\right)=2 f\left(\pi-\frac{\pi}{n}\right)-F(\pi), \\
& A_{e}=f\left(\frac{2 \pi\left[\frac{n}{2}\right]}{n}\right)=f(\pi) .
\end{aligned}
$$

Next we want to see which terms in the sum $A$ are positive and which are negative when $n \geqslant 473$. The sum $A$ can be written as

$$
A=\sum_{j=1}^{\left[\frac{n}{2}\right]-1} G\left(\frac{2 \pi j}{n}\right)
$$

where $G(x)=f(x)-F(x+\pi / n)$. Notice that the first term in the sum $A$ corresponds to $x=2 \pi / n$ and the last term corresponds to $x=\pi-3 \pi / n$ when $n$ is odd, and $x=\pi-2 \pi / n$ when $n$ is even. Let 
$I_{x}=[2 \pi / n, \pi-3 \pi / n]$ when $n$ is odd, and $I_{x}=[2 \pi / n, \pi-2 \pi / n]$ when $n$ is even. We claim that the function $G$ has a unique zero for $x \in I_{x}$ and this zero belongs to the interval $(\pi-5 \pi / n, \pi-4 \pi / n)$. Next we prove the claim.

To simplify the computations we introduce the change of variables $z=x+\pi / n$ and consider the equation $\widetilde{G}(z)=0$ with

$$
\widetilde{G}(z)=(f(z-\pi / n))^{2}-(F(z))^{2}=\frac{\alpha^{2}}{8\left(1-\sin \frac{\pi}{n} \sin z-\cos \frac{\pi}{n} \cos z\right)}-\frac{(\alpha-\cos z)^{2}}{\left(1+\alpha^{2}-2 \alpha \cos z\right)^{3}} .
$$

Let $g(z)$ be the numerator of the factorization of $\widetilde{G}(z)$, after doing the substitutions $\cos \frac{\pi}{n}=\alpha, \sin \frac{\pi}{n}=\sqrt{1-\alpha^{2}}$, and $\sin z=\sqrt{1-\cos ^{2} z}$ we get that equation $g(z)=0$ is equivalent to equation

$$
\begin{aligned}
& (1-\alpha)(1+\alpha)\left(\alpha^{2}\left(\alpha^{4}+4 \alpha^{2}+7\right)-2 \alpha\left(3 \alpha^{4}+9 \alpha^{2}+8\right) \cos z+\right. \\
& \left.4\left(3 \alpha^{4}+6 \alpha^{2}+2\right) \cos ^{2} z-8 \alpha\left(\alpha^{2}+1\right) \cos ^{3} z\right) \\
& =8 \sqrt{1-\alpha^{2}} \sqrt{1-\cos ^{2} z}(\alpha-\cos z)^{2}
\end{aligned}
$$

Squaring both sides of this equation to drop off the square roots and doing the substitution $c=\cos z$ we get an equation equivalent to $g(z)=0$ given by

$$
\begin{aligned}
\widetilde{g}(c)= & -\left(1-\alpha^{2}\right)\left(64\left(\alpha^{8}+\alpha^{6}-\alpha^{4}-\alpha^{2}-1\right) c^{6}-64 \alpha\left(3 \alpha^{8}+6 \alpha^{6}-\alpha^{4}-6 \alpha^{2}-6\right) c^{5}+\right. \\
& 16 \alpha^{2}\left(15 \alpha^{8}+45 \alpha^{6}+22 \alpha^{4}-42 \alpha^{2}-60\right) c^{4}- \\
& 16(\alpha-1) \alpha^{3}(\alpha+1)\left(10 \alpha^{6}+50 \alpha^{4}+95 \alpha^{2}+73\right) c^{3}+ \\
& 4 \alpha^{2}\left(15 \alpha^{10}+75 \alpha^{8}+133 \alpha^{6}+21 \alpha^{4}-168 \alpha^{2}+4\right) c^{2}- \\
& 4 \alpha^{3}\left(3 \alpha^{10}+18 \alpha^{8}+44 \alpha^{6}+30 \alpha^{4}-39 \alpha^{2}+8\right) c+ \\
& \left.\alpha^{4}\left(\alpha^{2}+3\right)\left(\alpha^{8}+4 \alpha^{6}+10 \alpha^{4}-4 \alpha^{2}+5\right)\right)=0 .
\end{aligned}
$$

Note that all solutions of equation $G(x)=G(z-\pi / n)=0$ are solutions of $\widetilde{g}(c)=0$ with $c=\cos z$.

The interval $I_{x}$ in the variable $c$ is given by $I_{c}=[\cos (3 \pi / n), \cos (\pi-$ $2 \pi / n)]$ when $n$ is odd and $I_{c}=[\cos (3 \pi / n), \cos (\pi-\pi / n)]$ when $n$ is even. In what follows we will work with $\alpha=\cos (\pi / n)$ instead of $n$, so we will not distinguish between $n$ odd and $n$ even and we take $I_{c}=[\cos (3 \pi / n), \cos (\pi-\pi / n)]$. Since $n=\pi / \arccos \alpha$, after some simplifications $I_{c}$ can be written as $I_{\alpha}=\left[4 \alpha^{3}-3 \alpha,-\alpha\right]$. We are interested in solutions of $\widetilde{g}(c)=0$ with $c \in I_{\alpha}$ and $\alpha \in[\cos (\pi / 473), 1) \subset D$ with $D=[999 / 1000,1)$. 
First we prove that the number of solutions of $\widetilde{g}(c)=0$ does not change for $\alpha \in D$. The number of solutions of $\widetilde{g}(c)=0$ can change when $\widetilde{g}(c)=0$ and $\widetilde{g}^{\prime}(c)=0$ simultaneously. We compute $\operatorname{Res}\left[\widetilde{g}(c), \widetilde{g}^{\prime}(c), c\right]$ and we get the following polynomial in the variable $\alpha$

$$
\begin{aligned}
T(\alpha)= & -4398046511104\left(\alpha^{2}-1\right)^{31} \alpha^{16}\left(\alpha^{8}+\alpha^{6}-\alpha^{4}-\alpha^{2}-1\right) \\
& \left(729 \alpha^{10}-2187 \alpha^{8}+459 \alpha^{6}+33831 \alpha^{4}+13824 \alpha^{2}-65536\right) .
\end{aligned}
$$

Applying Sturm's algorithm we see that there are no zeroes of $T$ for $\alpha \in D$. Therefore, using the properties of resultants, we get that the number of real solutions of $\widetilde{g}(c)=0$ does not change for $\alpha \in D$.

The number of solutions of $\widetilde{g}(c)=0$ with $c \in I_{\alpha}$ can change when $\alpha$ is such that either $c=4 \alpha^{3}-3 \alpha$ or $c=-\alpha$. Applying Sturm's algorithm we see that the polynomials $\widetilde{g}\left(4 \alpha^{3}-3 \alpha\right)$ and $\widetilde{g}(-\alpha)$ have no zeroes for $\alpha \in D$. So the number of solutions of $\widetilde{g}(c)=0$ with $c \in I_{\alpha}$ does not change for $\alpha \in D$. Applying Sturm's algorithm again we see that for instance for $\alpha=99999 / 100000 \in[\cos (\pi / 473), 1)$ there is a unique real root $c^{*}$ of $\widetilde{g}(c)=0$ with $c^{*} \in I_{\alpha}$. Next we see that this real root satisfies that $c^{*} \in(\cos (\pi-4 \pi / n), \cos (\pi-3 \pi / n))$. After some simplifications and taking $n=\pi / \arccos \alpha$ we get $\cos (\pi-3 \pi / n)=$ $3 \alpha-4 \alpha^{3}$ and $\cos (\pi-4 \pi / n)=-8 \alpha^{4}+8 \alpha^{2}-1$. By doing the change of variables $\gamma=(\alpha-99 / 100) /(1-\alpha)$ we get

$$
\widetilde{g}\left(3 \alpha-4 \alpha^{3}\right)=\frac{(100 \gamma+99)^{4}(200 \gamma+199)^{2}}{3814697265625 \cdot 10^{38}(\gamma+1)^{28}} B_{1},
$$

where $B_{1}$ is a polynomial of degree 20 in the variable $\gamma$ with positive coefficients that is given by

$$
\begin{aligned}
& \left(312500000000000000 \gamma^{10}+30078125000000000000 \gamma^{9}+130192968750000000000 \gamma^{8}+\right. \\
& 333726022656250000000 \gamma^{7}+560991664380859375000 \gamma^{6}+646166008553593750000 \gamma^{5}+ \\
& 516451807542245312500 \gamma^{4}+282813389281368250000 \gamma^{3}+101545293194930191250 \gamma^{2}+ \\
& 21586058638174381500 \gamma+2062870261351502751)\left(40625000000000000000 \gamma^{10}+\right. \\
& 401078125000000000000 \gamma^{9}+1781852968750000000000 \gamma^{8}+4691003022656250000000 \gamma^{7}+ \\
& 8104450691880859375000 \gamma^{6}+9601063173433593750000 \gamma^{5}+7898547219442445312500 \gamma^{4}+ \\
& 4455668938082168250000 \gamma^{3}+1649462704496130191250 \gamma^{2}+361845223038974381500 \gamma+ \\
& 35719897641551502751) .
\end{aligned}
$$


Since $\gamma>0$ for all $\alpha \in D, \widetilde{g}\left(3 \alpha-4 \alpha^{3}\right)>0$ for all $\alpha \in D$. In a similar way, by doing the change of variables $\gamma=\alpha-9 / 10$ we get

$$
\widetilde{g}\left(-8 \alpha^{4}+8 \alpha^{2}-1\right)=-\frac{(20 \gamma+19)^{6}}{3814697265625 \cdot 10^{16}(\gamma+1)^{34}} B_{2},
$$

where $B_{2}$ is a polynomial of degree 26 in the variable $\gamma$ with positive coefficients. Since $\gamma>0$ for all $\alpha \in D, \widetilde{g}\left(-8 \alpha^{4}+8 \alpha^{2}-1\right)<0$ for all $\alpha \in D$. Therefore the unique solution of $\widetilde{g}(c)$ with $c^{*} \in I_{\alpha}$ always satisfies that $c^{*} \in(\cos (\pi-4 \pi / n), \cos (\pi-3 \pi / n))$. This solution provides a solution $x^{*}$ of $G(x)=0$ with $x^{*} \in(\pi-5 \pi / n, \pi-4 \pi / n)$. This proves the claim.

Now we prove that $G(x)>0$ when $x \in I_{x}$ and $x<x^{*}$, and $G(x)<$ 0 when $x \in I_{x}$ and $x>x^{*}$. Since $f(x)>0$ and $F(x+\pi / n)>0$ for $x \in$ $I_{x}$ it is sufficient to analyze the sign of $\widetilde{G}(x)=(f(x))^{2}-(F(x+\pi / n))^{2}$. After some simplifications and using that $\alpha=\cos (\pi / n)$ we get

$$
\begin{aligned}
\widetilde{G}\left(\frac{2 \pi}{n}\right) & =\frac{\alpha^{2} g_{1}(\alpha)}{16\left(1-\alpha^{2}\right)\left(8 \alpha^{2}+1\right)^{3}}, \\
\widetilde{G}\left(\pi-\frac{4 \pi}{n}\right) & =-\frac{\alpha^{2}\left(1-\alpha^{2}\right) g_{2}(\alpha)}{16\left(2 \alpha^{2}-1\right)^{2}\left(8 \alpha^{4}-5 \alpha^{2}+1\right)^{3}},
\end{aligned}
$$

where

$$
\begin{aligned}
& g_{1}(\alpha)=512 \alpha^{6}+192 \alpha^{4}+24 \alpha^{2}-255 \\
& g_{2}(\alpha)=512 \alpha^{10}-448 \alpha^{8}-680 \alpha^{6}+1003 \alpha^{4}-434 \alpha^{2}+63 .
\end{aligned}
$$

After doing the change of variables $\gamma=\alpha-99 / 100$ in the functions $g_{1}$ and $g_{2}$ respectively we get that $g_{1}(\gamma+99 / 100)$ and $g_{2}(\gamma+99 / 100)$ are polynomials in the variable $\gamma$ of degrees 6 and 10 respectively having positive coefficients, so $g_{1}(\gamma+99 / 100)>0$ and $g_{2}(\gamma+99 / 100)>0$ in $D$ because $\gamma>0$ in $D$. Moreover $8 \alpha^{4}-5 \alpha^{2}+1>0$ for all $\alpha$. Therefore $\widetilde{G}(2 \pi / n)>0$ and $\widetilde{G}(\pi-4 \pi / n)<0$ for all $\alpha \in[\cos (\pi / 473), 1)$. In short, $G(x)>0$ when $x \in I_{x}$ and $x<x^{*}$, and $G(x)<0$ when $x \in I_{x}$ and $x>x^{*}$

We have just seen that $A$ can be written as $A=A_{p}+A_{m}$ with 
$A_{p}>0$ and $A_{m}<0$ where

$$
\begin{aligned}
A_{p}=A_{p o} & =\sum_{j=1}^{\left[\frac{n}{2}\right]-2}\left[f\left(\frac{2 \pi j}{n}\right)-F\left(\frac{2 \pi j}{n}+\frac{\pi}{n}\right)\right], \\
A_{m}=A_{m o} & =f\left(\frac{2 \pi\left(\left[\frac{n}{2}\right]-1\right)}{n}\right)-F\left(\frac{2 \pi\left(\left[\frac{n}{2}\right]-1\right)}{n}+\frac{\pi}{n}\right) \\
& =f\left(\pi-\frac{3 \pi}{n}\right)-F\left(\pi-\frac{2 \pi}{n}\right),
\end{aligned}
$$

when $n$ is odd, and

$$
\begin{aligned}
A_{p}=A_{p e}= & \sum_{j=1}^{\left[\frac{n}{2}\right]-3}\left[f\left(\frac{2 \pi j}{n}\right)-F\left(\frac{2 \pi j}{n}+\frac{\pi}{n}\right)\right] \\
A_{m}=A_{m e}= & f\left(\frac{2 \pi\left(\left[\frac{n}{2}\right]-1\right)}{n}\right)-F\left(\frac{2 \pi\left(\left[\frac{n}{2}\right]-1\right)}{n}+\frac{\pi}{n}\right)+ \\
& f\left(\frac{2 \pi\left(\left[\frac{n}{2}\right]-2\right)}{n}\right)-F\left(\frac{2 \pi\left(\left[\frac{n}{2}\right]-2\right)}{n}+\frac{\pi}{n}\right) \\
= & f\left(\pi-\frac{2 \pi}{n}\right)-F\left(\pi-\frac{\pi}{n}\right)+f\left(\pi-\frac{4 \pi}{n}\right)-F\left(\pi-\frac{3 \pi}{n}\right),
\end{aligned}
$$

when $m$ is even.

In short, $H(n)=2 A_{p o}+2 A_{m o}+A_{o}$ when $n$ is odd and $H(n)=$ $2 A_{p e}+2 A_{m e}+A_{e}$ when $m$ is even, where the terms $A_{p o}$ and $A_{p e}$ are positive. Next we see that the terms $2 A_{m o}+A_{o}$ and $2 A_{m e}+A_{e}$ are also positive. We start with the case $n$ odd. Since $\alpha+1>4 \alpha^{2}-3 \alpha+1$, after some simplifications we see that for all $\alpha \in[\cos (\pi / 473), 1)$

$$
\begin{aligned}
2 A_{m o}+A_{o} & =2 f\left(\pi-\frac{3 \pi}{n}\right)-2 F\left(\pi-\frac{2 \pi}{n}\right)+2 f\left(\pi-\frac{\pi}{n}\right)-F(\pi) \\
& =\frac{1}{\sqrt{\alpha+1}}\left(\frac{\sqrt{2} \alpha^{2}}{2 \alpha-1}-\frac{2(2 \alpha-1)}{\left(4 \alpha^{2}-3 \alpha+1\right)^{3 / 2}}-\frac{1}{(\alpha+1)^{3 / 2}}\right) \\
& >\frac{1}{\sqrt{\alpha+1}}\left(\frac{\sqrt{2} \alpha^{2}}{2 \alpha-1}-\frac{4 \alpha-1}{\left(4 \alpha^{2}-3 \alpha+1\right)^{3 / 2}}\right) .
\end{aligned}
$$

After doing the change of variables $\gamma=\alpha-9 / 10$

$$
\left(\frac{\sqrt{2} \alpha^{2}}{2 \alpha-1}\right)^{2}-\left(\frac{4 \alpha-1}{\left(4 \alpha^{2}-3 \alpha+1\right)^{3 / 2}}\right)^{2}=\frac{B_{3}}{800(5 \gamma+2)^{2}\left(200 \gamma^{2}+210 \gamma+77\right)^{3}},
$$


where $B_{3}$ is a polynomial of degree 10 in the variable $\gamma$ with positive coefficients, so it is positive because $\gamma>0$ for $\alpha \in[\cos (\pi / 473), 1)$. In short $2 A_{m o}+A_{o}>0$ for all $\alpha \in[\cos (\pi / 473), 1)$ and consequently if $n$ is odd, then $H(n)>0$ for all $n \geqslant 473$.

In the case $n$ even

$2 A_{m e}+A_{e}=2 f\left(\pi-\frac{2 \pi}{n}\right)-2 F\left(\pi-\frac{\pi}{n}\right)+2 f\left(\pi-\frac{4 \pi}{n}\right)-2 F\left(\pi-\frac{3 \pi}{n}\right)+f(\pi)$.

It is easy to check that $f(x), F(x)>0$ for all $x \in(2 \pi / n, \pi)$. After doing the change of variables $\gamma=(\alpha-9 / 10) /(1-\alpha)$ we have

$$
\begin{aligned}
& \left(2 f\left(\pi-\frac{4 \pi}{n}\right)\right)^{2}-\left(2 F\left(\pi-\frac{\pi}{n}\right)\right)^{2}=\frac{\alpha^{2}}{4\left(2 \alpha^{2}-1\right)^{2}}-\frac{16 \alpha^{2}}{\left(3 \alpha^{2}+1\right)^{3}} \\
& =\frac{25(\gamma+1)^{2}(10 \gamma+9)^{2}(20 \gamma+19)}{4\left(50 \gamma^{2}+80 \gamma+31\right)^{2}\left(400 \gamma^{2}+740 \gamma+343\right)^{3}} \\
& \left(1120000 \gamma^{4}+4184000 \gamma^{3}+5836000 \gamma^{2}+3601080 \gamma+829053\right) .
\end{aligned}
$$

and

$$
\begin{gathered}
\left(2 f\left(\pi-\frac{2 \pi}{n}\right)+f(\pi)\right)^{2}-\left(2 F\left(\pi-\frac{3 \pi}{n}\right)\right)^{2}=\frac{1}{16}(\alpha+2)^{2}-\frac{16 \alpha^{2}\left(2 \alpha^{2}-1\right)^{2}}{\left(8 \alpha^{4}-5 \alpha^{2}+1\right)^{3}} \\
=\frac{B_{4}}{1600(\gamma+1)^{2}\left(10000 \gamma^{4}+34500 \gamma^{3}+44575 \gamma^{2}+25570 \gamma+5497\right)^{3}},
\end{gathered}
$$

where $B_{4}$ is a polynomial of degree 14 in $\gamma$ with positive coefficients, so they are positive for $\alpha \in[\cos (\pi / 473), 1)$ because $\gamma$ is positive in $[\cos (\pi / 473), 1)$. In short if $n$ is even, $H(n)>0$ for all $n \geqslant 473$. This completes the proof.

Lemma 4 Let $N_{m}(a, n)=a_{12} b_{2}-a_{22} b_{1}$, then the following statements hold

(a) $N_{m}(1 / \sqrt{3}, n)>0$ for $3 \leqslant n<53$ and $N_{m}(1 / \sqrt{3}, n)<0$ for $n \geqslant 53$,

(b) $N_{m}\left(a^{*}, n\right)>0$ for $n=3,4, N_{m}\left(a^{*}, n\right)<0$ for $5 \leqslant n<53$ and $N_{m}\left(a^{*}, n\right)>0$ for $53 \leqslant n<473$,

(c) $\lim _{a \rightarrow 0^{+}} N_{m}(a, n)=+\infty$ for $n \geqslant 473$.

\section{Proof.}

If $a=1 / \sqrt{3}$, then from Lemma 2 (d) $a_{12}=0$, and consequently $N_{m}(1 / \sqrt{3}, n)=-a_{22} b_{1}$. Since $\alpha \in[1 / 2,1)$ (recall that $\alpha=\cos (\pi / n)$ ), 
we get $1 / \sqrt{3}>\alpha / \sqrt{3}$; then from Lemma 2 (e) $a_{22}>0$. Moreover from Lemma 2 (b) and (f) $b_{1}<0$ for $n<53$ and $b_{1}>0$ for $53 \leqslant n \leqslant 473$ and from Lemma 2 (c) $b_{1}>0$ for $n \geqslant 473$. This proves statement (a).

Statement (b) can be proved by computing the values of $N_{m}\left(a^{*}, n\right)$ for $n=3, \ldots, 472$.

Now we prove statement (c). If follows easily that

$$
\lim _{a \rightarrow 0^{+}} N_{m}(a, n)=\lim _{a \rightarrow 0^{+}} \frac{1}{4 a^{3}}\left(\alpha b_{1}-b_{2}\right),
$$

Then the proof follows from the fact that $\alpha b_{1}-b_{2}>0$ for all $n \geqslant 473$ (see Lemma 3).

\subsection{Proof of Theorem 1 for $n=4$}

We are interested in the solutions of system (14) with $m, \mu>0$. When $n=4$ we claim that we can find a solution of system (14) with $\mu=0$ and $m>0$ that can be continued to a family of solutions of (14) with $\mu>0$ small and $m>0$.

Now we prove the claim. When $n=4$ the coefficients of system (14) are

$$
\begin{aligned}
a_{11} & =-\frac{8 \sqrt{2}}{\left(2 a^{2}+1\right)^{3 / 2}}+2 \sqrt{2}+\frac{6 \sqrt{2}}{5 \sqrt{5}} \\
a_{12} & =\frac{2}{\left(a^{2}+1\right)^{3 / 2}}-\frac{1}{4 a^{3}} \\
a_{21} & =-\frac{8}{\left(2 a^{2}+1\right)^{3 / 2}}+\sqrt{2}+\frac{1}{2}, \\
a_{22} & =\frac{4}{\left(2 a^{2}+1\right)^{3 / 2}}-\frac{1}{4 \sqrt{2} a^{3}}, \\
b_{1} & =-\frac{4}{\left(a^{2}+1\right)^{3 / 2}}+\frac{1}{\sqrt{2}}+\frac{1}{4} \\
b_{2} & =\frac{8}{5 \sqrt{5}}-\frac{2 \sqrt{2}}{\left(a^{2}+1\right)^{3 / 2}} .
\end{aligned}
$$

We assume that $a_{11} \neq 0$ and we look for solutions of (14) with $\mu=0$. Under these assumptions, from (14) we get $m=-b_{1} / a_{11}$. Straightforward computations show that $a_{11}>0$ for $a>a^{\star, 1}=$ $\sqrt{\frac{5}{58} \sqrt[3]{3886-870 \sqrt{5}}-\frac{1}{2}} \approx 0.7584732493, a_{11}<0$ for $a<a^{\star, 1}$, and 
$a_{11}=0$ for $a=a^{\star, 1}$; and $b_{1}>0$ for $a>a^{\star, 2}=\sqrt{4\left(\frac{2}{7}\right)^{2 / 3} \sqrt[3]{9-4 \sqrt{2}}-1} \approx$ 1.262765225, is $b_{1}<0$ for $a<a^{\star, 2}$, and $b_{1}=0$ for $a=a^{\star, 2}$. Therefore $m>0$ when $a \in\left(a^{\star, 1}, a^{\star, 2}\right)$. On the other hand, from (14) again, $\mu=0$ when $N_{\mu}(a)=a_{21} b_{1}-a_{11} b_{2}=0$. We see that $N_{\mu}\left(a^{\star, 1}\right)>0$ and $N_{\mu}\left(a^{\star, 2}\right)<0$. Therefore, since $N_{\mu}$ is defined for all $a \in \mathbb{R}^{+}$, there exists at least a zero $a=a_{\mu}$ of $N_{\mu}$ with $a_{\mu} \in\left(a^{\star, 1}, a^{\star, 2}\right)$. If this zero does not satisfy condition $D\left(a_{\mu}\right)=a_{11} a_{22}-a_{21} a_{12}=0$, then this solution can be continued to a family of solutions of (14) with $m>0$ and $\mu>0$ small, which is given by

$m=m(a)=\frac{N_{m}(a)}{D(a)}=\frac{a_{12} b_{2}-a_{22} b_{1}}{a_{11} a_{22}-a_{12} a_{21}}, \quad \mu=\mu(a)=\frac{N_{\mu}(a)}{D(a)}=\frac{a_{21} b_{1}-a_{11} b_{2}}{a_{11} a_{22}-a_{12} a_{21}}$.

If the value $a_{\mu}$ is such that $D\left(a_{\mu}\right)=0$, then either system (14) has no solutions when $N_{m}\left(a_{\mu}\right)=a_{12} b_{2}-a_{22} b_{1} \neq 0$, or it has infinitely many solutions when $N_{m}\left(a_{\mu}\right)=0$. We note that the first case is not possible because, since $a_{11} \neq 0$, from condition $D\left(a_{\mu}\right)=0$ we get $a_{22}=a_{21} a_{12} / a_{11}$ and from condition $N_{\mu}\left(a_{\mu}\right)=0$ we get $b_{2}=$ $b_{1} a_{21} / a_{11}$, then $N_{m}\left(a_{\mu}\right)=0$. The infinitely many solutions that we can have when $N_{\mu}\left(a_{\mu}\right)=0, D\left(a_{\mu}\right)=0$ and $N_{m}\left(a_{\mu}\right)=0$ are

$$
m=\frac{-b_{1}-a_{12} \mu}{a_{11}}, \quad \mu \in \mathbb{R} .
$$

In this last case, since $-b_{1} / a_{11}>0$ there exists infinitely many families of solutions of (14) with $m>0$ and $\mu>0$ small.

Notice that if $a_{11}=0$, that is $a=a^{\star, 1}$, then the solution is $m=m\left(a^{\star, 1}\right) \approx 28614318,2.4304510854826634604$ and $\mu=\mu\left(a^{\star, 1}\right) \approx$ $2.4304510854 \neq 0$ (see (15)). This completes the proof of the claim, so Theorem 1 is proved for $n=4$.

Remark: We can eliminate the square roots of equation $N_{\mu}(a)=0$ by squaring conveniently as many times as we need, then dropping off the denominators we get a polynomial equation of degree 24 in the variable $a$ whose set of solutions contains at least all solutions of equation $N_{\mu}(a)=0$. This polynomial equation has two positive real solutions that can be computed numerically but only $a=a_{\mu} \approx$ 1.1778268479 is a solution of $N_{\mu}(a)=0$. Proceeding in the same way with the equation $D(a)=0$ we arrive to a polynomial equation of degree 36 in the variable $a$ that has also two positive real solutions but only $a=a_{d} \approx 0.7111336024$ is a solution of $D(a)=0$. Therefore $N_{\mu}$ and $D$ are not zero simultaneously, so for all positive $a$ with $a \neq a_{d}$ 
system (14) has a unique solution $m=m(a), \mu=\mu(a)$ which is given by (15), and for $a=a_{d}$ system (14) has no solution. The solution $m=m(a), \mu=\mu(a)$ provides a central configurations when $m(a)>0$ and $\mu(a)>0$. Now we find the set where these conditions are satisfied.

As above we eliminate the square roots of equation $N_{m}(a)=0$, we drop off the denominators we get a polynomial equation of degree 36 in the variable $a$ that has 6 real solutions but only the solutions $a=a_{m, 1} \approx 0.1092983002, a=a_{m, 2} \approx 1.3587461717$ and $a=a_{m, 3}=$ 3.7016660737 are solutions of $N_{m}(a)=0$. Analyzing the signs of $N_{\mu}$, $D$ and $N_{m}$ we get that $N_{\mu}(a)>0$ for $a \in\left(0, a_{\mu}\right)$ and $N_{\mu}(a)<0$ for $a \in\left(a_{\mu},+\infty\right) ; D(a)>0$ for $a \in\left(a_{d},+\infty\right)$ and $D(a)<0$ for $a \in\left(0, a_{d}\right)$; and $N_{m}(a)>0$ for $a \in\left(a_{m, 1}, a_{m, 2}\right) \cup\left(a_{m, 3},+\infty\right)$ and $N_{m}(a)<0$ for $a \in\left(0, a_{m, 1}\right) \cup\left(a_{m, 2}, a_{m, 3}\right)$. So $m(a)>0$ and $\mu(a)>0$ for $a \in\left(a_{d}, a_{\mu}\right)=(0.7111336024,1.1778268479)$ and the configuration is central.

\subsection{Proof of Theorem 1 for $n \geqslant 5$}

Proceeding in a similar way than in the case $n=4$, we claim that for all $n \geqslant 5$ we can find at least a value of $a$ for which there exist a solution of (14) with $m=0$ and $\mu>0$ that can be continued to a family of solutions of (14) with $m>0$ and $\mu>0$. This family of solutions will provide a family of central configuration of our problem, this proves Theorem 1 for $n \geqslant 5$. Next we prove the claim.

Let

$N_{m}(a, n)=a_{12} b_{2}-a_{22} b_{1}, \quad N_{\mu}(a, n)=a_{21} b_{1}-a_{11} b_{2}, \quad D(a, n)=a_{11} a_{22}-a_{21} a_{12}$.

We assume that $a_{12} \neq 0$ and we look for solutions of (14) with $m=0$. If $m=0$ and $a_{12} \neq 0$, then from (14) we get $\mu=-b_{1} / a_{12}$. We analyze the sign of $-b_{1} / a_{12}$. From Lemma 2 , if $5 \leqslant n<53$, then $-b_{1} / a_{12}>0$ for $a \in\left(1 / \sqrt{3}, a^{*}\right)$, if $53 \leqslant n \leqslant 472$, then $-b_{1} / a_{12}>$ 0 for $a \in\left(a^{*}, 1 / \sqrt{3}\right)$; and if $n \geqslant 473$, then $-b_{1} / a_{12}>0$ for $a \in$ $(0,1 / \sqrt{3})$. Now we prove that there exists at least a value of $a$ such that $m=0$ in the region where $\mu>0$. If $m=0$, then $N_{m}(a, n)$ must be zero. From Lemma 4 we have that if $5 \leqslant n \leqslant 472$, then $N_{m}(1 / \sqrt{3}, n)$ and $N_{m}\left(a^{*}, n\right)$ have different signs, and if $n \geqslant 473$ then $\lim _{a \rightarrow 0^{+}} N_{m}(a, n)=+\infty$ and $N_{m}(1 / \sqrt{3}, n)<0$. Since $N_{m}(a, n)$ is well defined for all $a \in \mathbb{R}^{+}$we get that for all $n \geqslant 5$ there exist at least a value of $a, a=a_{m}$, such that $N_{m}\left(a_{m}, n\right)=0$ and $\mu>0$. Notice that if $a_{12}=0$ (i.e. $a=1 / \sqrt{3}$, see Lemma 2$)$ then $N_{m}(1 / \sqrt{3}, n)$ is not zero, 
so the assumption $a_{12} \neq 0$ is not restrictive. If $D\left(a_{m}, n\right) \neq 0$, then the solution of (14) wit $a=a_{m}$ is unique and it can be continued to a family of solutions of (14) in $a$ with $m>0$ and $\mu>0$. As in (15), this family is given by $m=N_{m}(a, n) / D(a, n)$ and $\mu=N_{\mu}(a, n) / D(a, n)$. If the value $a_{m}$ is such that $D\left(a_{m}, n\right)=0$, then either system (14) has no solutions when $N_{\mu}\left(a_{m}, n\right) \neq 0$, or it has infinitely many solutions when $N_{\mu}\left(a_{m}, n\right)=0$. We note that the first case is not possible. This is proved following the same arguments than in the case $n=4$.

The infinitely many solutions that we have when $N_{m}\left(a_{m}, n\right)=0$, $D\left(a_{m}, n\right)=0$ and $N_{\mu}\left(a_{m}, n\right)=0$ are

$$
m \in \mathbb{R}, \quad \mu=\frac{-b_{1}-a_{11} m}{a_{12}} .
$$

In this last case since $-b_{1} / a_{12}>0$, for all $n \geqslant 5$ there exists infinitely many families of solutions of (14) with $m>0$ and $\mu>0$. This proves the claim, so Theorem 1 is proved for $n \geqslant 5$.

Remark: We have computed numerically the values of $a$ for which $N_{m}(a, n)=0$ and the values of $a$ for which $D(a, n)=0$ for $5 \leqslant n \leqslant 600$ and they never coincide. Moreover for $5 \leqslant n \leqslant 600$ we have found that both functions, $N_{m}(a, n)$ and $D(a, n)$, have a unique positive zero. Therefore we have strong numerical evidences that for each $n \geqslant 5$ system (14) cannot have infinitely many solutions and that there exists a unique value of $a$ such that the solution of system (14) satisfies that $m=0$ and $\mu>0$.

Proceeding as in the case $n=4$ we have computed for $5 \leqslant n \leqslant 600$ the set where the solution of system (14) provides a central configuration; that is, $m>0$ and $\mu>0$. Let $S$ denote this set. We see that for all $5 \leqslant n \leqslant 600$ the set $S$ is an interval whose endpoints are the solutions of $N_{m}(a, n)=0$ and $D(a, n)=0$, respectively. In particular, if $a=a_{m}(n)$ is the solution of $N_{m}(a, n)=0$ and $a=a_{d}(n)$ is the solution of $D(a, n)=0$, then $S=\left(a_{d}(n), a_{m}(n)\right)$ when $n \leqslant 128$ and $S=\left(a_{m}(n), a_{d}(n)\right)$ when $n>128$. The functions $a_{m}(n)$ and $a_{d}(n)$ for $5 \leqslant n \leqslant 600$ are plotted in Figure 1 (a). In Figure 1 (b) we plot the length of the interval $S$ for $5 \leqslant n \leqslant 600$. In Table 1 we give the numerical value of $S$ form some values of $n$. 


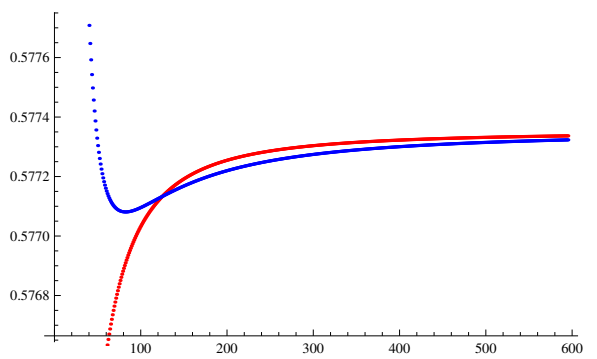

(a)

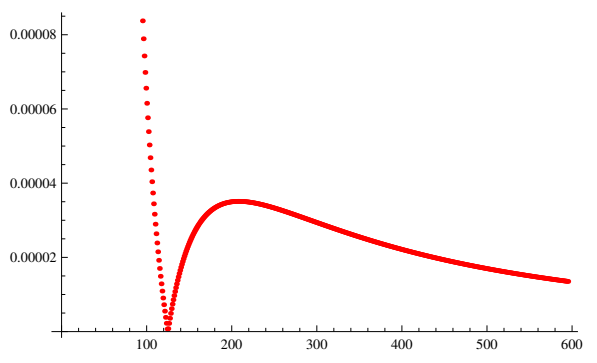

(b)

Figure 1: (a) The plot of the solution of $N_{m}(a, n)=0, a_{m}(n)$, (the function having a minimum) and the plot of the solution of $D(a, n)=0, a_{d}(n)$, (the increasing function). (b) The plot of the length of the interval $S$.

\begin{tabular}{|c|c|}
\hline$n$ & $D$ \\
\hline 5 & $(0.6054508601,1.047660644)$ \\
6 & $(0.5735628097,0.8966193846)$ \\
7 & $(0.5649527372,0.7993129461)$ \\
8 & $(0.5631917539,0.7341595533)$ \\
9 & $(0.5635643266,0.6904238044)$ \\
10 & $(0.5645832304,0.6608539922)$ \\
11 & $(0.5657382437,0.6405062783)$ \\
12 & $(0.5668499893,0.6261710814)$ \\
13 & $(0.5678603234,0.6158181455)$ \\
\hline \hline$n$ & $D$ \\
\hline 128 & $(0.5771316623,0.5771323567)$ \\
129 & $(0.5771338677,0.5771346567)$ \\
200 & $(0.5772516235,0.5772167740)$ \\
300 & $(0.5773027503,0.5772730689)$ \\
400 & $(0.5772997096,0.5773220724)$ \\
500 & $(0.5773143684,0.5773314948)$ \\
600 & $(0.5773233276,0.5773368180)$ \\
\hline
\end{tabular}

\begin{tabular}{|c|c|}
\hline$n$ & $D$ \\
\hline 20 & $(0.5722706230,0.5876432515)$ \\
30 & $(0.5746983627,0.5798882468)$ \\
40 & $(0.5757077735,0.5780266218)$ \\
50 & $(0.5762252724,0.5774202950)$ \\
60 & $(0.5765274024,0.5771954491)$ \\
70 & $(0.5767199392,0.5771108814)$ \\
80 & $(0.5768506065,0.5770839197)$ \\
90 & $(0.5769435911,0.5770820288)$ \\
100 & $(0.5770122548,0.5770911638)$ \\
\hline
\end{tabular}

Table 1: The interval $S$ providing central configurations for some $n \geqslant 5$. 


\section{Proof of statement (a) of Theorem 1}

When $n=3$ the coefficients of system (14) are

$$
\begin{aligned}
a_{11} & =-\frac{3}{\left(a^{2}+\frac{1}{4}\right)^{3 / 2}}+\frac{4}{\sqrt{3}}+\frac{4}{9}, \\
a_{12} & =\frac{2}{\left(a^{2}+1\right)^{3 / 2}}-\frac{1}{4 a^{3}}, \\
a_{21} & =\frac{4}{\sqrt{3}}-\frac{3}{2\left(a^{2}+\frac{1}{4}\right)^{3 / 2}}, \\
a_{22} & =\frac{1}{\left(a^{2}+\frac{1}{4}\right)^{3 / 2}}-\frac{1}{8 a^{3}}, \\
b_{1} & =\frac{1}{\sqrt{3}}-\frac{3}{\left(a^{2}+1\right)^{3 / 2}}, \\
b_{2} & =\frac{4}{9}-\frac{3}{2\left(a^{2}+1\right)^{3 / 2}} .
\end{aligned}
$$

Recall that

$N_{m}(a)=a_{12} b_{2}-a_{22} b_{1}, \quad N_{\mu}(a)=a_{21} b_{1}-a_{11} b_{2}, \quad D(a)=a_{11} a_{22}-a_{12} a_{21}$.

Assuming that $D(a) \neq 0$, the solutions of system (14) are $m=m(a)=$ $N_{m}(a) / D(a)$ and $\mu=\mu(a)=N_{\mu}(a) / D(a)$.

Next we see that there are no solutions with $m(a)>0$ and $\mu(a)>$ 0 , therefore there are not central configurations for our problem when $n=3$.

Let $a=a_{m}$ denote a solution of $N_{m}(a)=0, a=a_{\mu}$ a solution of $N_{\mu}(a)=0$ and $a=a_{d}$ a solution of $D(a)=0$. The set of $a$ 's where $m(a)>0$ and $\mu(a)>0$ is formed by an interval or a union of intervals whose endpoints are zeroes of either $N_{m}, N_{\mu}$ or $D$. In particular, we have the following possibilities for the endpoints of each interval: two zeroes of $N_{m}$; two zeroes of $N_{\mu}$; a zero of $N_{m}$ and a zero of $D$; a zero of $N_{\mu}$ and a zero of $D$; and two zeroes of $D$.

First we prove that there is no $a$ such that $m=0$ and $\mu>0$. If $m=0$ then

$$
\mu=-\frac{b_{1}}{a_{12}}=-\frac{b_{2}}{a_{22}} .
$$

Straightforward computations show that $b_{1}>0$ for $a>\sqrt{2}, b_{1}=0$ for $a=\sqrt{2}$, and $b_{1}<0$ for $0<a<\sqrt{2} ; a_{12}>0$ for $a>1 / \sqrt{3}$, 
$a_{12}=0$ for $a=1 / \sqrt{3}$, and $a_{12}<0$ for $a<1 / \sqrt{3} ; b_{2}>0$ for $a>$ $\sqrt{5} / 2, b_{2}=0$ for $a=\sqrt{5} / 2$, and $b_{2}<0$ for $a<\sqrt{5} / 2$; and finally $a_{22}>0$ for $a>1 /(2 \sqrt{3}), a_{22}=0$ for $a=1 /(2 \sqrt{3})$, and $a_{22}<0$ for $a<1 /(2 \sqrt{3})$. Therefore $\mu>0$ (that is, $-b_{1} / a_{12}>0$ and $-b_{2} / a_{22}>0$ simultaneously) when $1 / \sqrt{3}<a<\sqrt{5} / 2$. Now we prove that equation $N_{m}(a)=0$ has no solutions with $a \in(1 / \sqrt{3}, \sqrt{5} / 2)$. We eliminate the square roots of equation $N_{m}(a)=0$ by squaring conveniently as many times as we need, then dropping off the denominators we get a polynomial equation $P_{m}(a)=0$ of degree 36 in the variable $a$. We do the change of variables $\gamma=-(5(2 a-1)) /(2(5 a-6))$ in the polynomial $P_{m}(a)$ (notice that $\gamma>0$ for all $a \in(1 / \sqrt{3}, \sqrt{5} / 2)$ ) and we get a rational function in the variable $\gamma$ having all the coefficients positive, therefore there is no solution of system (14) with $m=0$ and $\mu>0$.

In a similar way, if $\mu=0$ then

$$
m=-\frac{b_{1}}{a_{11}}=-\frac{b_{2}}{a_{21}} .
$$

Straightforward computations show that $a_{11}<0$ for $a<\bar{a}$ with

$$
\bar{a}=\frac{1}{2} \sqrt{9\left(\frac{2}{1+3 \sqrt{3}}\right)^{2 / 3}-1} \approx 0.8992976476,
$$

$a_{11}=0$ for $a=\bar{a}$, and $a_{11}<0$ for $a<\bar{a} ; b_{2}>0$ for $a>\sqrt{5} / 2$, $b_{2}=0$ for $a=\sqrt{5} / 2$, and $b_{2}<0$ for $a<\sqrt{5} / 2$; and finally $a_{21}>0$ for $a>1 / \sqrt{2}, a_{22}=0$ for $a=1 / \sqrt{2}$, and $a_{22}<0$ for $a<1 / \sqrt{2}$. Therefore $m>0$ (that is, $-b_{1} / a_{11}>0$ and $-b_{2} / a_{21}>0$ simultaneously) when $\bar{a}<a<\sqrt{5} / 2$. By eliminating the square roots of equation $N_{\mu}(a)=$ 0 and dropping off the denominators we get a polynomial equation $P_{\mu}(a)=0$ of degree 24 in the variable $a$. Doing the change of variables $\gamma=(4-5 a) /(5 a-6)$ in the polynomial $P_{\mu}(a)$ (notice that $\gamma>0$ in $\bar{a}<a<\sqrt{5} / 2)$ as above we see that this polynomial equation has no solutions in the interval $\bar{a}<a<\sqrt{5} / 2$. Therefore there is no solution of system(14) with $\mu=0$ and $m>0$.

Now we prove that $D$ and $N_{\mu}$ (respectively, $N_{m}$ ) are not zero simultaneously. We eliminate the square roots of equation $N_{\mu}(a)=0$ (respectively, $N_{m}(a)=0$ ) by squaring conveniently as many times as we need, then dropping off the denominators we get a polynomial equation $P_{\mu}(a)=0$ of degree 24 (respectively, $P_{m}(a)=0$ of degree 36 ). Doing the same with equation $D(a)=0$ we get a polynomial equation 
$P_{D}(a)=0$ of degree 36 . We do the resultant of the polynomials $P_{D}$ and $P_{\mu}$, and of $P_{D}$ and $P_{m}$ with respect to $a$, and we get two numbers different from zero. Then by the properties of the resultants these two pairs of polynomials cannot have a common zero.

Finally we prove that the set of $a$ 's where $m(a)>0$ and $\mu(a)>0$ cannot be formed by an interval whose endpoints are both zeroes $D$. Since $D(1 /(2 \sqrt{3}))$ is different from zero we can assume that $a_{22} \neq 0$, then from $D(a)=0$ we obtain $a_{11}=a_{12} a_{21} / a_{22}$. Substituting this value of $a_{11}$ into the expressions of $N_{m}(a)$ and $N_{\mu}(a)$ we get

$$
N_{m}(a)=-a_{22} b_{1}+a_{12} b_{2}, \quad N_{\mu}(a)=\frac{a_{21}}{a_{22}}\left(a_{22} b_{1}-a_{12} b_{2}\right) .
$$

If $a_{21} / a_{22}>0$ then $N_{m}$ and $N_{\mu}$ have different signs, so in this case the zeroes of $D$ cannot be the endpoints of an interval providing central configurations. Straightforward computations show that $a_{21} / a_{22}>0$ when either $0<a<1 /(2 \sqrt{3})$ or $a>1 / \sqrt{2}$. On the other hand, by doing the change of variables $\gamma=$ we see that the polynomial $P_{D}$ has no zeroes in the interval $a \in[1 /(2 \sqrt{3}), 1 / \sqrt{2}]$, and since this interval is the unique set where $N_{m}$ and $N_{\mu}$ have the same sign, it follows that two zeroes of $D$ never can be the endpoints of an interval providing central configurations.

Remark. Computing numerically the positive real solutions of $N_{m}(a)=0, N_{\mu}(a)=0$ and $D(a)=0$ as we have done for $n=4$ we see that $N_{m}(a)=0$ has a unique real solution with $a>0$, $a=a_{m} \approx 0.13809757 ; N_{\mu}(a)=0$ has two real solutions with $a>0$, $a=a_{\mu, 1} \approx 0.3019817428$ and $a=a_{\mu, 2} \approx 2.5508746364$; and $D(a)=0$ has a unique real solution with $a>0 a=a_{d} \approx 0.1235465602$. Then Analyzing the signs of $N_{m}, B_{\mu}$ and $D$ we get that $N_{m}(a)<0$ for $a \in\left(0, a_{m}\right)$ and $N_{m}(a)>0$ for $a \in\left(a_{m},+\infty\right) ; N_{\mu}(a)<0$ for $a \in\left(a_{\mu, 1}, a_{\mu, 2}\right)$ and $N_{\mu}(a)>0$ for $a \in\left(0, a_{\mu, 1}\right) \cup\left(a_{\mu, 2},+\infty\right)$; and $D(a)<0$ for $a \in\left(a_{d},+\infty\right)$ and $D(a)>0$ for $a \in\left(0, a_{d}\right)$. Therefore $m>0$ for $a \in\left(a_{d}, a_{m}\right)$, and $\mu>0$ for $a \in\left(0, a_{d}\right) \cup\left(a_{\mu, 1}, a_{\mu, 2}\right)$. In short, there is no positive $a$ such that $\mu>0$ and $m>0$.

\section{Acknowledgements}

Both authors are partially supported by the Ministerio de Ciencia, Innovación y Universidades, Agencia Estatal de Investigación grants 
MTM2016-77278-P (FEDER). The second author is also partially supported by the Agència de Gestió d'Ajuts Universitaris i de Recerca grant 2017SGR1617, and the H2020 European Research Council grant MSCA-RISE-2017-777911.

\section{References}

[1] E. Barrabés and J.M. Cors, On strictly convex central configurations of the 2n-body problem, J. Dyn. Diff. Eq., https://doi.org/10.1007/s10884-018-9708-5

[2] F. Cedó and J. Llibre, Symmetric central configurations of the spatial n-body problem, J. of Geometry and Physics 6 (1989), 367394.

[3] K.C. Chen and J.S. Hsiao, Convex central configurations of the $n$-body problem which are not strictly convex, J. Dyn. Diff. Equ. 24(2012), 119-128.

[4] M. Corbera and J. Llibre, On the existence of bi-pyramidal central configurations of the n+2-body problem with an n-gon basis, Cont. Dyn. Discrete Systems 33 (2013), 1049-1060.

[5] N. Faycal, On the classification of pyramidal central configurations. Proc. Amer. Math. Soc. 124 (1996), 249-258.

[6] A.C. Fernandes, B.A. Garcia and L.F. Mello, Convex but not strictly convex central configurations, J. Dynam. Differential Equations 30 (2018), 1427-1438.

[7] M. Gidea and J. Llibre, Symmetric planar central configurations of five bodies: Euler plus two, Celest. Mech. Dyn. Astron. 106 (2010), 89-107.

[8] X. Liu, On double pyramidal central configuration with parallelogram base. Xinan Shifan Daxue Xuebao Ziran Kexue Ban 26 (2001), 521-525.

[9] X. Liu, Double pyramidal central configurations with a concave quadrilateral base. J. Chongqing Univ. 1 (2002), 67-69.

[10] X. Liu, A class of double pyramidal central configurations of 7body with concave pentagon base. Xinan Shifan Daxue Xuebao Ziran Kexue Ban 27 (2002), 494-496. 
[11] X. Liu, Existence and uniqueness for a class of double pyramidal central configurations with a concave pentagonal base. J. Chongqing Univ. 2 (2003), 28-30.

[12] X. Liu and X. Chen, Double pyramidal central configurations of 5-bodies with arbitrary triangle base. Sichuan Daxue Xuebao 40 (2003), 190-194.

[13] X. Liu, Existence and uniqueness of a class of double pyramidal central configurations in six-body problems. J. Chongqing Univ. 3 (2004), 97-101.

[14] X. Liu, X. Du and T. Feng, Existence and uniqueness for a class of nine-bodies central configurations. J. Chongqing Univ. 5 (2006), $53-56$.

[15] L.F. Mello and A.C. Fernandes, New spatial central configurations in the 5-body problem. An. Acad. Bras. Ciênc. (2010, accepted for publication).

[16] L.F. Mello and A.C. Fernandes, New classes of spatial central configurations for the $n+3$ body problem. Nonlinear Anal. Real World Appl. 12 (2011), 723-730.

[17] R. Moeckel and C. Simó, Bifurcation of spatial central configurations from planar ones. SIAM J. Math. Anal. 26 (1995), 978-998.

[18] T. Ouyang, Z. Xie, and S. Zhang, Pyramidal central configurations and perverse solutions, Electronic Journal of Differential Equations, 106 (2004), 1-9.

[19] D. Yang and S. Zhang, Necessary conditions for central configurations of six-body problems. Southeast Asian Bull. Math. 27 (2003), 739-747.

[20] S. Zhang and Q. Zhou, Double pyramidal central configurations. Phys. Lett. A 281 (2001), 240-248. 